\title{
Indicadores de erosividad de la lluvia en una cuenca de la Serranía del Litoral Central de Venezuela'
}

\section{Rainfall erosivity indicators in a watershed of the Central Coast Mountain Range of Venezuela}

\author{
Williams Méndez ${ }^{2}$ (1) , Henry Pacheco ${ }^{3}$ (1), Loan Landaeta ${ }^{4}$ (1), \\ Edgar Menéndez ${ }^{5}$ (), Miguel Pérez ${ }^{6}$ (1), Gypsi Parra ${ }^{7}$ (1) y Jessiré Fuentes ${ }^{8}$
}

\begin{abstract}
RESUMEN
Uno de los principales problemas ambientales atribuidos al uso de la tierra en la cuenca del río Chichiriviche (estado Vargas, Venezuela), está vinculado con la actividad agrícola que, origina condiciones para la activación de procesos erosivos en el suelo, frente al impacto de la lluvia. Bajo este contexto, se analizaron el potencial erosivo y las intensidades extremas de las precipitaciones, como indicadores de erosividad en la cuenca montañosa del río Chichiriviche. Se analizaron la distribución espacio-temporal de las precipitaciones, la frecuencia de los eventos extremos de las intensidades y se estimaron los Índices de Fournier Anual, Modificado de Fournier, Concentración de las Precipitaciones, Erosividad Total y Potencial Erosivo de las Lluvias. La distribución espacial de las Iluvias está controlada principalmente por la altitud; su régimen anual se caracteriza por una mayor concentración de precipitaciones a finales de año. Las intensidades máximas oscilaron entre $56,43 \mathrm{~mm} / \mathrm{h}$ y $81,95 \mathrm{~mm} / \mathrm{h}$ para eventos de 1 hora de duración y 100 años de período de retorno. Los índices estimados, indicaron bajo a moderado potencial erosivo de las lluvias. La ocurrencia de procesos erosivos en el área estudiada, está relacionada con la intensidad de las precipitaciones extraordinarias como eventos puntuales aislados.
\end{abstract}

Palabras clave: potencial erosivo, eventos extremos, erosividad, cuenca de drenaje, ambientes montañosos.

Ingreso: 2019-04-06 / Aceptado: 2020-06-24

Departamento de Construcciones Civiles, Facultad de Ciencias Matemáticas Físicas y Químicas, Universidad Técnica de Manabí, Portoviejo 130105, Manabí, Ecuador. Centro de Investigación Estudios del Medio Físico Venezolano, Instituto Pedagógico de Caracas, Universidad Pedagógica Experimental Libertador, El Paraíso 1020, Caracas, Distrito Capital, Venezuela. E-mail: williamsmendez@gmail.com

Departamento de Ingeniería Agrícola, Facultad de Ingeniería Agrícola, Universidad Técnica de Manabí, Lodana 13132, Santa Ana, Manabí, Ecuador. E-mail: henrypacheco@gmail.com

Centro de Investigación Estudios del Medio Físico Venezolano, Instituto Pedagógico de Caracas, Universidad Pedagógica Experimental Libertador, El Paraíso 1020, Caracas, Distrito Capital, Venezuela. E-mail: profesor_loan1@hotmail.com

Departamento de Construcciones Civiles, Facultad de Ciencias Matemáticas Físicas y Químicas, Universidad Técnica de Manabí, Portoviejo 130105, Manabí, Ecuador. E-mail: edfamenendez@gmail.com

Departamento de Ciencias de la Tierra, Instituto Pedagógico de Caracas, Universidad Pedagógica Experimental Libertador, El Paraíso 1020, Caracas, Distrito Capital, Venezuela. E-mail: miguelleonardogod@hotmail.com

Departamento de Ciencias de la Tierra, Instituto Pedagógico de Caracas, Universidad Pedagógica Experimental Libertador, El Paraíso 1020, Caracas, Distrito Capital, Venezuela. E-mail:mariselita07@gmail.com

Departamento de Ciencias de la Tierra, Instituto Pedagógico de Caracas, Universidad Pedagógica Experimental Libertador, El Paraíso 1020, Caracas, Distrito Capital, Venezuela. E-mail: jessirefm@hotmail.com 


\begin{abstract}
One of the main environmental problems attributed to land use in the Chichiriviche river watershed (Vargas state, Venezuela) is linked to agricultural activity, which causes conditions that trigger erosive processes in the soil due to the impact of the rainfall. The erosive potential and extreme rainfall intensities were analyzed as indicators of erosivity on the Chichiriviche River mountainous watershed. The spatio-temporal rainfall distribution and extreme event frequency were analyzed. The Indices of Annual Fournier, Modified Fournier, Rainfall Concentration, Total Erosivity and the Rain Erosive Potential. The spatial distribution of rainfall is controlled by the altitude. Its annual regime is characterized by a greater concentration of rainfall at year end. The maximum intensities ranged between $56.43 \mathrm{~mm} / \mathrm{h}$ and $81.95 \mathrm{~mm} / \mathrm{h}$ for events of 1 hour and 100 -year return period. The indices indicated low to moderate rainfall erosive potential. The occurrence of erosive processes on the studied area is more related to extraordinary rainfall as isolated punctual events.
\end{abstract}

Keywords: erosive potential, extreme events, erosivity, watershed, mountainous environments.

\title{
Introducción
}

La susceptibilidad del medio físico a la ocurrencia de procesos erosivos en una cuenca montañosa depende de las condiciones del relieve, así como del clima, tipo de suelo, cobertura vegetal, drenaje y uso de la tierra (Castelán et al., 2014). Sin embargo, la génesis de dichos procesos se vincula principalmente al impacto de la lluvia como elemento detonante (Angulo y Beguería, 2013). La erosión como fenómeno natural o inducido por la intervención antrópica, genera cuantiosas pérdidas económicas anuales, así como materiales y humanas (Ponvert et al., 2007), éstas últimas sobre todo con la ocurrencia de eventos de procesos de remoción en masa (deslizamientos y flujos de detritos) de magnitudes importantes.

Uno de los principales problemas ambientales que se relacionan con el uso de la tierra en la cuenca del río Chichiriviche, está vinculado con la actividad agrícola (cultivos altamente erosivos como los hortícolas) que se desarrolla sobre terrenos con pendientes que oscilan entre $15 \%$ y $35 \%$, sin ningún tipo de práctica conservacionista contra la erosión del suelo. Esta situación conlleva, a su vez, a otro problema atribuido a los procesos erosivos, ya que la eliminación de la vegetación nativa, así como la remoción y mecanización del suelo por actividades agrícolas y pecuarias, generan una mayor exposición de su superficie al impacto de las lluvias (Flórez, 2018).

El análisis espacio-temporal del potencial erosivo y de las intensidades de eventos extremos de la lluvia, es de importancia para el diseño de medidas preventivas, mitigantes y de control contra los impactos de éstas sobre los medios físico y socio-económico, así como para la adecuación de los usos y prácticas agrícolas. En el estudio del potencial erosivo de la lluvia, ha sido de amplia utilidad a nivel mundial el desarrollo de ecuaciones e índices que evalúan tales rasgos de las precipitaciones, para cuantificar y cualificar el impacto de éstas en la pérdida del suelo.

Un número importante de trabajos han realizado contribuciones significativas en el desarrollo de métodos para estimar la agresividad y la erosividad anual de la lluvia, así como para el análisis de la variabilidad espacio-temporal del potencial erosivo de las lluvias y, las relaciones de la variabilidad temporal de la erosividad de la lluvia con el ENOS y el Cambio Climático (Duan et al., 2016; 
Hoomehr et al., 2016; Meshesha et al., 2016; Panagos et al., 2016; Qin et al., 2016; Xie et al., 2016; Ballabio et al., 2017; Chen y Zha, 2017; Panagos et al., 2017; García-Barrón et al., 2018).

Otros trabajos, han realizado aportes importantes en el estudio de los eventos extremos de precipitación, centrando sus esfuerzos en tópicos de investigación orientados hacia: (a) relaciones entre el ENOS y otros fenómenos climáticos con EEP (Limsakul y Singhruck, 2016), (b) estacionariedad y no estacionariedad de los EEP (Gao et al., 2016; Gu et al., 2017), (c) variabilidad espacial y temporal de la FEEP (Gao et al., 2017), (d) incertidumbre en el análisis de FEEP (Hailegeorgis y Alfredsen, 2017), (e) índices de EEP (Santos et al., 2017), (f) análisis de tendencia en EEP (Wang et al., 2017); (g) umbrales de EEP (Douka y Karacostas, 2018) y (h) nuevos modelos para la interpretación de EEP (Pakalidou y Karacosta, 2018).

Considerando la incidencia de las precipitaciones como activadoras de procesos erosivos, aunado a los impactos que ocasionan estos últimos sobre el medio humano, es objetivo de esta investigación, analizar el potencial erosivo y las intensidades extremas de las precipitaciones como indicadores de erosividad en la cuenca montañosa del río Chichiriviche. Esta investigación aporta elementos que permiten identificar relaciones entre los índices que evalúan el potencial erosivo de las lluvias, las intensidades de eventos extremos de éstas y la activación de procesos erosivos, así como, cual de estos análisis representa un mejor indicador de erosividad de las lluvias en el área.

\section{Área de estudio}

La cuenca del río Chichiriviche está situada geográficamente en la Región Centro Norte del territorio venezolano (sector occidental del Estado Vargas) definida por las siguientes coordena-

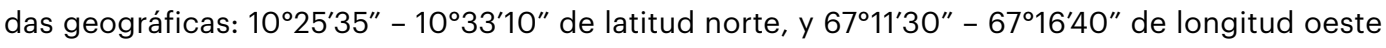
(figura 1). Esta cuenca ocupa una superficie aproximada de $76,02 \mathrm{~km}^{2}$. Forma parte de la provincia orográfica Cordillera de la Costa (Sistema Montañoso del Caribe) en su tramo central. El relieve local es dominado por el extremo oriental de la vertiente norte de la serranía de Rancho Grande (ramal litoral de la Cordillera de la Costa) (Guevara, 1983), la cual termina abruptamente hacia el mar en una costa de inmersión (Arismendi, 2007). El relieve se distingue por presentar una topografía abrupta y escarpada típica de un sector montañoso, con laderas de fuertes pendientes superiores a $45 " \%$ y alturas entre 0 msnm (línea de costa) y 2280 msnm (Pico Geremba o El Palmar) (figura 2).

La litología está constituida por rocas metamórficas de las fajas tectónicas Asociación Metamórfica Ávila: Augengneis de Peña de Mora y Complejo Metamórfico San Julián; Asociación Meta-Sedimentaria Caracas: Esquisto de Las Brisas; y Asociación Metamórfica La Costa: Anfibolita de Nirgua; y por los depósitos fluvio-torrenciales de fondo de valle en el tramo final del río Chichiriviche (Urbani y Rodríguez, 2004; Urbani et al., 2006). Los perfiles de meteorización son poco profundos en las laderas de muy fuertes pendientes, lo cual facilita la rápida saturación de los mismos en presencia de eventos de precipitaciones extraordinarias (Amundaray, 2000; Grases et al., 2000). Ello conlleva el desarrollo de cárcavas profundas, deslizamientos y derrumbes, así como severas restricciones para el desarrollo de infraestructuras (Ministerio del Ambiente y de los Recursos Naturales Renovables [MARNR], 1983). 
Figura $\mathrm{N}^{\circ} 1$

Localización del área de estudio (cuenca del río Chichiriviche, estado Vargas, Venezuela) y sectores intervenidos por la actividad agrícola y con mayor exposición a los procesos erosivos

(cuencas media y alta, tonalidades de verdes más claros).

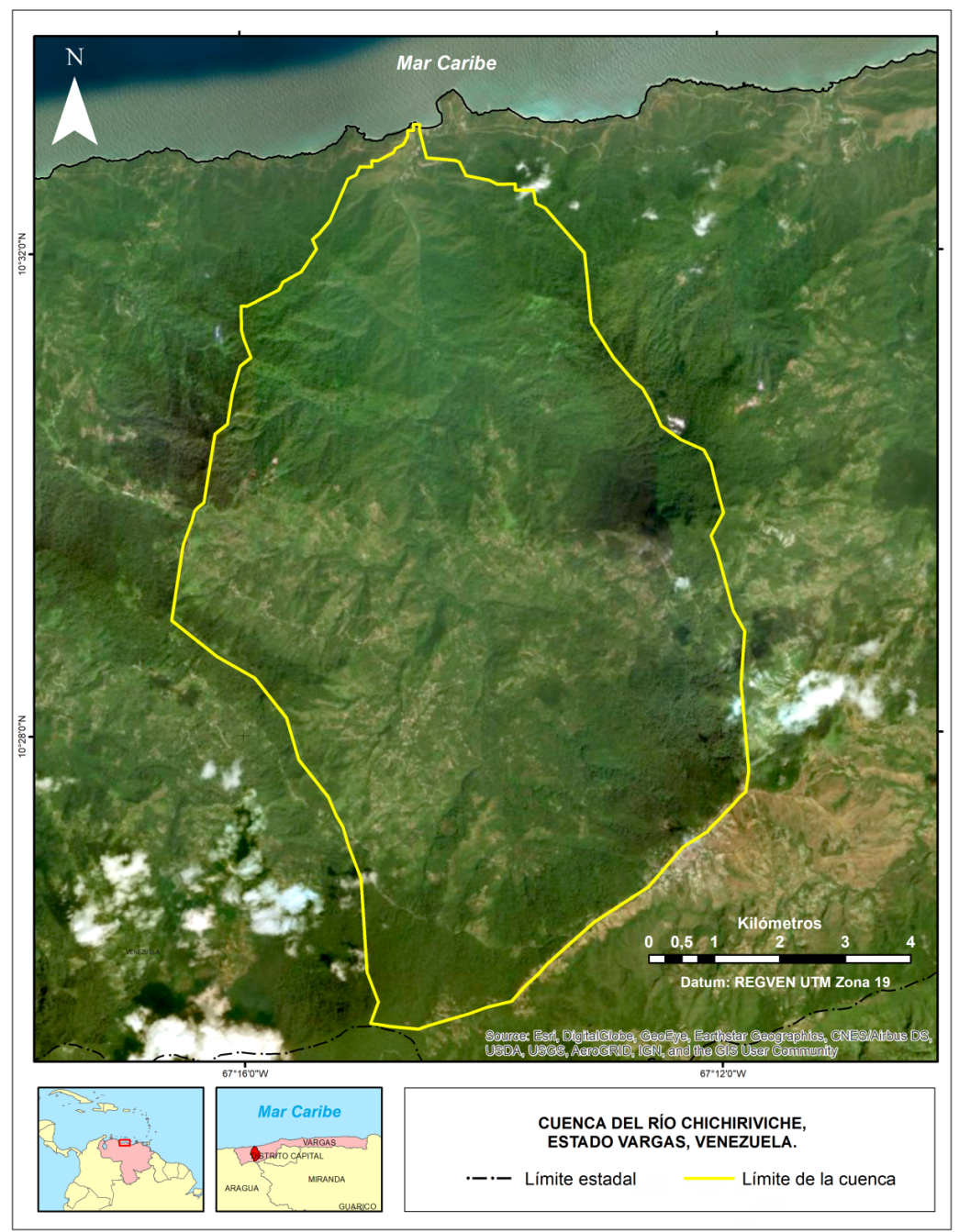

Fuente: Esri et al. (2019).

El clima se caracteriza por presentar temperaturas medias anuales que oscilan entre $19,9^{\circ} \mathrm{C}$ y 21,2 ${ }^{\circ} \mathrm{C}$ (Estación Climatológica Carayaca) (Ministerio del Poder Popular para el Ambiente [MPPA], 2011). Las precipitaciones son escasas con promedios anuales que superan los $500 \mathrm{~mm}$ (entre 555 mm y 1251,6 mm) (MPPA, 2011). El sector está enmarcado en un tipo climático Bshi (Clima Tropical Estepario) para la parte baja (franja litoral), y Aw (Clima Tropical de Sabana) para las partes media y alta (sector montañoso) de acuerdo con la clasificación de Köppen (Foghin, 2002).

El drenaje de la cuenca está constituido por el curso principal del río Chichiriviche (recorrido de 16,84 km en sentido sur-norte), en el cual vierten sus aguas afluentes de régimen permanente, 
Figura $\mathrm{N}^{\circ} 2$

Vista 3D de la cuenca del río Chichiriviche (estado Vargas, Venezuela) en la que se aprecia su topografía abrupta, escarpada y de fuertes pendientes, característica del relieve montañoso (serranía de Rancho Grande) sobre el que se emplaza.

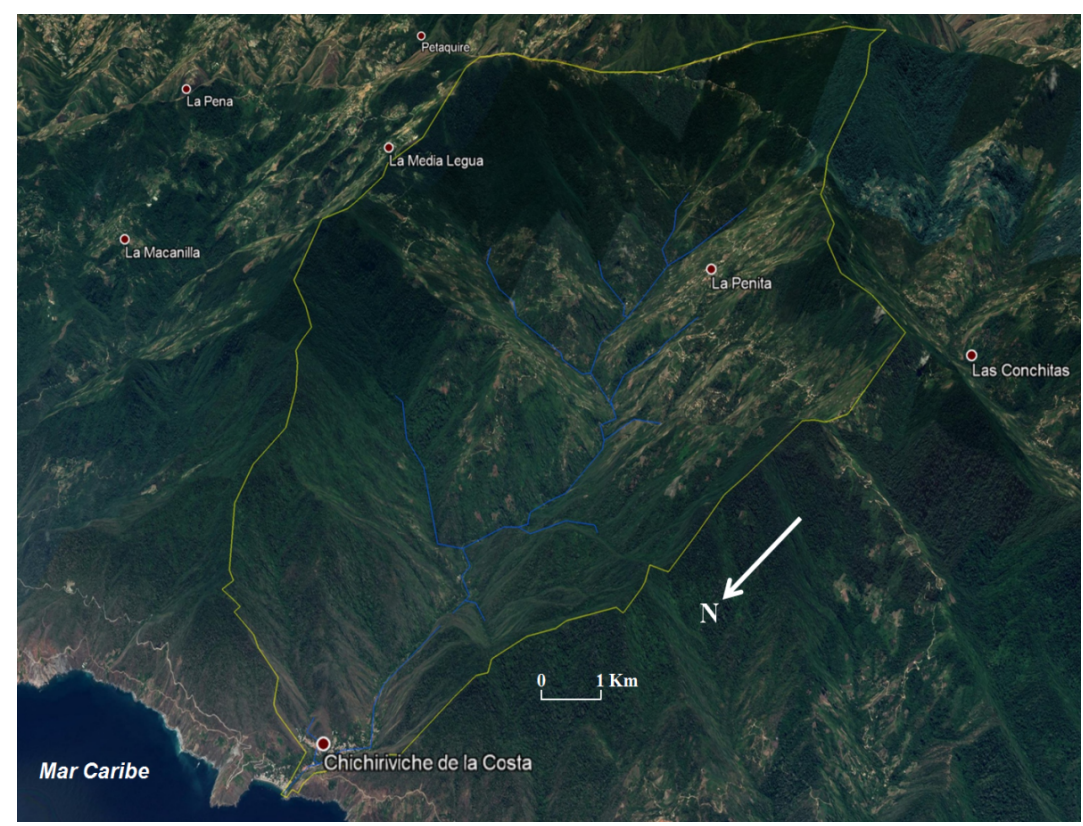

Fuente: Google Earth (2019).

así como otros numerosos de carácter intermitente. Desde la línea de costa hasta la fila montañosa, se observan las formaciones vegetales: litoral, cardonales y espinares, bosque deciduo, bosque semi-deciduo, bosque transicional, bosque de galería y bosque húmedo (Steyermark y Huber, 1978; Amend, 1991; Petróleos de Venezuela, S. A., 1993). Las actividades económicas en el área se concentran principal y mayoritariamente en la agricultura (figura 1). La mayor parte de la población es rural.

\section{Material y métodos}

\section{Información pluviométrica}

Se compilaron los registros pluviométricos (totales mensuales y anuales y láminas máximas anuales de precipitación) para un período de veinticinco años (1956-1980), correspondientes a siete estaciones pluviográficas (Puerto Cruz, La Guitarrita, La Peñita, Alto de Ño León, Puerto Oricao, Puerto Chichiriviche y Hacienda Naranjal) y una climatológica (Carayaca) ubicadas dentro de la jurisdicción del estado Vargas (Venezuela), y con influencia sobre la cuenca de drenaje del río Chichiriviche. La información fue suministrada por el Instituto Nacional de Meteorología e Hidrología (INAMEH), publicada por el Ministerio del Poder Popular para el Ambiente [MPPA] (2015) a través de sus direcciones generales de Cuencas y de Hidrología y Meteorología. 
Como tratamiento previo de las series temporales de precipitación (totales mensuales y anuales), se desagregaron los datos englobados y se estimaron los faltantes por medio de los Métodos Racional y Sustitución por el Promedio (Guevara, 1987). Para el caso de las láminas máximas anuales de precipitación se eliminaron los datos que generaban ruido estadístico en las series, mediante la aplicación de la Prueba de Datos Dudosos (U. S. Water Resources Council, 1981).

\section{Distribución espacio-temporal de las lluvias}

Para dicha caracterización se construyeron histogramas con los datos correspondientes a los promedios mensuales (pluviogramas) de cada una de las estaciones consideradas para el análisis. Con base en estos pluviogramas se describieron los regímenes o distribución de las lluvias a lo largo del año, tomando como criterios el comportamiento modal de la distribución de los promedios mensuales, los picos de máximas y mínimas, la definición de períodos o estaciones, los porcentajes aproximados de las principales concentraciones de las lluvias en determinados espacios temporales del año y, los factores locales y posibles causas meteorológicas tropicales y/o extratropicales que condicionan la génesis y distribución de las lluvias en el área de estudio.

\section{Análisis de frecuencia de eventos extremos}

Se realizó un análisis de frecuencia de las profundidades de láminas máximas anuales de precipitación para distintas duraciones de la lluvia y distintos períodos de retorno, mediante el ajuste de la distribución de probabilidades de Valores Extremos Tipo I (Gumbel, 1941) según el método de Kite (1977), que contempla estimar la probabilidad de excedencia $(P(X \geq X))$, la probabilidad de no excedencia $(P(X<x))$, el período de retorno $\left(T_{R}\right)$, la variable reducida $\left(y_{T R}\right)$, el factor de frecuencia $\left(K_{T R}\right)$ y el ajuste Gumbel propiamente dicho, mediante la siguiente expresión:

$$
x_{T_{R}}=X_{m}+K_{T_{R}} S_{x}
$$

Donde $X_{m}$ y $S_{x}$ representan a la media $(\mathrm{mm})$ y a la desviación estándar $(\mathrm{mm})$ respectivamente, correspondientes a los valores de profundidades de láminas máximas anuales de precipitación de la serie histórica. Por último, se realizó también el ajuste para períodos de retorno preestablecidos de 2, 5, 10, 25, 50, 100, 500 y 1000 años, lo cual permitió estimar eventos extremos desde menores frecuencias hasta frecuencias más amplias. Luego se calculó el coeficiente de correlación entre los valores de profundidades de láminas máximas anuales de precipitación de la serie histórica y los valores simulados por el modelo Gumbel, para verificar la confiabilidad y/o bondad del ajuste correspondiente a cada duración de la lluvia.

Posteriormente, se calcularon las intensidades máximas anuales de precipitación para distintas duraciones de la lluvia, y para los períodos de retorno preestablecidos anteriormente. A su vez, estos valores de intensidad se ajustaron y/o suavizaron, por medio de la aplicación de la ecuación de intensidad de la lluvia de Wenzel (1982):

$$
i=\left[100\left(\frac{\frac{c}{T_{d}^{e}+f}-i_{0}}{i_{0}}\right)\right]^{2}
$$


Donde $i$ es la intensidad máxima anual de precipitación $(\mathrm{mm} / \mathrm{h})$ para una duración de la lluvia y un período de retorno dado, $T_{d}$ es la duración de la lluvia ( $h$ ), c, e y $f$ son coeficientes que varían con el lugar y el período de retorno, e $i_{o}$ es la intensidad máxima anual de precipitación $(\mathrm{mm} / \mathrm{h})$ para la misma duración de la lluvia y el mismo período de retorno dado, obtenida sin la aplicación de la ecuación de Wenzel. Esta ecuación se aplicó para calibrar los valores de los coeficientes c, e y $\mathrm{f}$. Se recalcularon las intensidades máximas anuales de precipitación aplicando nuevamente la ecuación de Wenzel, de la manera como se expresa:

$$
i=\frac{c}{T_{d}^{e}+f}
$$

A continuación, estos valores de intensidades máximas anuales de precipitación se transformaron nuevamente en profundidades de láminas máximas anuales de precipitación. Con estos datos se construyeron las curvas de profundidad-duración-frecuencia (PDF) y de intensidad-duración- frecuencia (IDF).

\section{Índices del potencial erosivo de las precipitaciones}

Para diversos escenarios geográficos a nivel mundial, se han estimado con frecuencia el Índice de Fournier Anual (IFA) (Fournier, 1960), Índice Modificado de Fournier (IMF) (Arnoldus, 1980), Índice de Concentración de las Precipitaciones (ICP), Índice de Erosividad Total (IET) y el Índice del Potencial Erosivo de las Lluvias (R) (Wischmeier, 1959). Estos métodos están basados en la energía cinética de las gotas de lluvia, fundamental para estimar la erosión por impacto (Angulo y Beguería, 2013) y, son ampliamente conocidos y utilizados a nivel mundial.

Para el análisis del potencial erosivo de las precipitaciones en este trabajo, se estimaron cinco índices mediante el desarrollo de ocho procedimientos (uno para el IFA, dos para el IMF (IMF $\mathrm{F}_{1}$ $\left.I \mathrm{IF}_{2}\right)$, dos para el ICP $\left(\mathrm{ICP}_{1}\right.$ e ICP $)$, dos para el IET (IET $\left.\mathrm{e} \mathrm{IET}_{2}\right)$, y uno para el R). En el caso de los índices $\mathrm{IMF}_{1}, \mathrm{ICP}_{1}$ e IET $\mathrm{I}_{1}$, sus estimaciones se realizaron a partir de los promedios mensuales del período de lluvias considerado en el estudio y, para el caso de los índices IMF ${ }_{2}, I C P_{2}$ e IET ${ }_{2}$, se realizaron a partir de los totales mensuales y anuales de cada uno de los años de la serie.

\section{Resultados y discusión}

\section{Espacio-temporalidad de las lluvias}

El Estado Vargas se configura como un espacio de contrastes climáticos. La diferencia altitudinal y la orientación de la serranía del Litoral respecto a la circulación de vientos y perturbaciones provenientes del noreste del Caribe y de Los Llanos venezolanos constituyen los principales factores y elementos que condicionan el clima de la cuenca.

La distribución de las precipitaciones en la cuenca corresponde con el modelo de inversión estacional propia de la zona de Caribe con la ocurrencia del invierno astronómico para el hemisferio norte en los últimos meses del año. La diferencia de calor solar durante estos meses, condiciona el desplazamiento meridional de la Convergencia Intertropical, dejando el norte vene- 
zolano bajo la acción de los vientos alisios (cinturón subtropical del hemisferio norte), situaciones frontales $y$, perturbaciones o inestabilidades atmosféricas como tormentas tropicales, ondas del este y vaguadas.

El recorrido de los alisios paralelos a la costa, genera condiciones atmosféricas y oceanográficas que dificultan el ascenso de las masas de aire $y$, por tanto, la formación de nubosidad y precipitaciones (Vila, 1960; Foghin, 2002). Sin embargo, a finales de año, cuando provienen desde el norte y se introducen debajo de las masas de aire caliente continental, se deshacen en abundantes precipitaciones sobre el margen costero y la orografía litoral.

En la cuenca y sus proximidades, se identifican dos tipos de comportamientos pluviométricos (figura 3):

(a) En la franja litoral y cuenca baja, la precipitación oscila entre $555 \mathrm{~mm}$ y 665,3 mm anuales (estaciones Puerto Oricao, Puerto Chichiriviche y Puerto Cruz) y se distribuye de manera casi uniforme durante todo el año; por otra parte, se observa un ligero incremento de los montos totales anuales de precipitación en sentido este - oeste, en la franja litoral. Si bien se identifica una disminución de las precipitaciones entre enero y marzo ( $12,5 \%$ a $14,0 \%$ del total anual) y precipitaciones abundantes a finales de año, entre octubre y diciembre $(33,4 \%$ a $35,3 \%)$, en esta uniformidad pluviométrica también se evidencia la ocurrencia de tres picos de máximas en los meses de mayo, agosto y octubre-noviembre y, un mínimo principal en el mes de febrero, definiendo un régimen de distribución anual de las precipitaciones de tipo irregular. En general, los montos totales y promedios mensuales y anuales de lluvia en este sector del área de estudio, así como su patrón de distribución temporal a lo largo del año, indican escasas precipitaciones (ningún mes del año supera los 100 mm, a excepción de los meses agosto y octubre en la estación Carayaca y, en algunas estaciones, no se supera los $80 \mathrm{~mm}$ mensuales), definiendo un contexto climático de tipo semi-árido.

(b) En las cuencas media y alta, las lluvias superan los $800 \mathrm{~mm}$ anuales hasta alcanzar los 1251,6 mm (estaciones Carayaca, Alto de Ño León, La Peñita, Hacienda Naranjal y La Guitarrita), y tienen dos períodos bien definidos y contrastantes respecto a la cuenca baja: un período corto de escasas precipitaciones entre diciembre y marzo (14,3\% a 14,9\% anual) y un período de lluvias más largo desde abril, concentrándose entre los meses de agosto a noviembre $(47,5 \%$ a $51,8 \%$ anual), denotando un régimen pluviométrico estacional de tipo bimodal, que se caracteriza por presentar dos picos de máximas, una primera que oscila entre abril y junio y, una segunda más pronunciada entre agosto y octubre, mientras que, el mínimo principal, se ubica en el mes de febrero. De igual manera, se observa un incremento importante de los montos anuales de precipitación (entre $40 \mathrm{~mm}$ y $100 \mathrm{~mm}$ ) en sentido este - oeste. Los montos totales y promedios mensuales y anuales de lluvia en estos sectores de la cuenca, así como su patrón de distribución temporal a lo largo del año, indican precipitaciones más abundantes (entre 7 y 8 meses del año, durante la estación lluviosa de abril a noviembre están por encima de los $80 \mathrm{~mm}$ ), definiendo un contexto climático de tipo semi-húmedo.

Es evidente que la orografía litoral constituye un factor con una marcada influencia en la distribución altitudinal de las precipitaciones, determinando una variación pluviométrica (totales anuales) ascendente desde la línea de costa (estación Puerto Chichiriviche, $22 \mathrm{msnm}$ ) hasta la fila montañosa, caracterizada por: un incremento del $45 \%$ a los 408 msnm (estación Hacienda 
Figura $\mathrm{N}^{\circ} 3$

Distribución espacio-temporal de las precipitaciones en la cuenca del río Chichiriviche (estado Vargas, Venezuela).

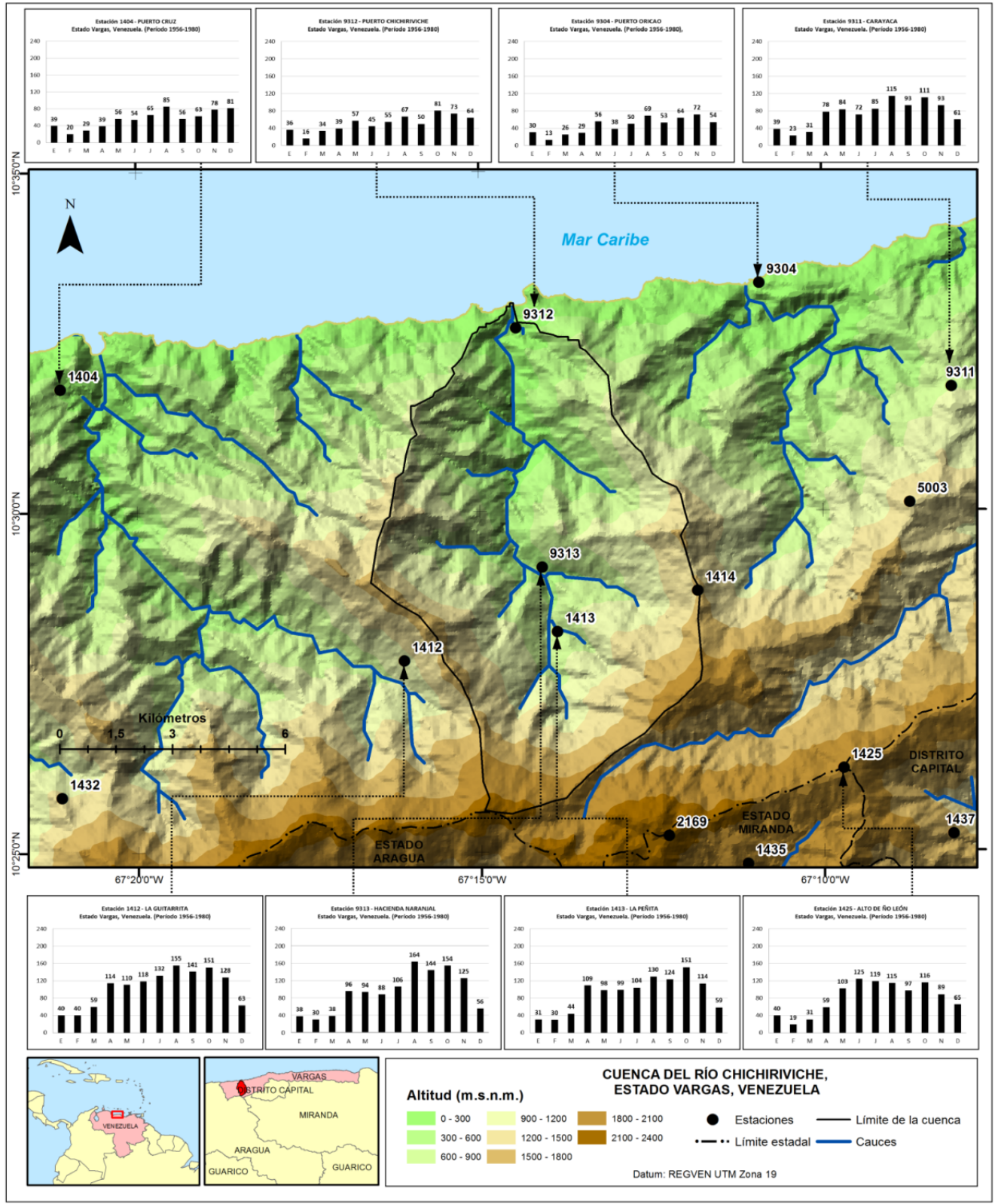

Fuente: Elaboración propia.

Naranjal), seguido de un ligero descenso de un 4\% a los 644 msnm (estación La Peñita) y, posteriormente, de otra disminución de un 10\% a los 2101 msnm (estación Alto de Ño León). Este comportamiento indica que, la mayor descarga anual de las precipitaciones, ocurre entre la cuenca media y su transición hacia la cuenca alta. 


\section{Eventos extremos de precipitación}

Según González y Córdova (2006), una de las tareas más importantes en el estudio de las lluvias máximas del Litoral Central venezolano lo constituye el poder establecer con cierto grado de confiabilidad, las intensidades máximas de la lluvia tanto para duraciones menores de 60 minutos -que son las que controlan los picos de los hidrogramas de las crecidas de las cuencas del litoralcomo para las lluvias de varios días que están, posiblemente, asociadas a los grandes deslaves.

Se aprecia que para 1 hora (60 min) de duración de la lluvia, las láminas máximas anuales son de magnitudes significativas, que oscilan desde 26,90 $\mathrm{mm}$ (Puerto Oricao), para un período de retorno de 2 años, hasta $111,21 \mathrm{~mm}$ (Puerto Cruz) para un período de retorno de 1000 años, con lo cual es de esperarse que eventos de precipitaciones concentradas y con montos entre los valores referidos, para 1 hora de duración de la lluvia, generen la activación de procesos erosivos. Esto cobra mayor significado, sobre todo para eventos de períodos de retorno de 50, 100 y 500 años (figura 4).

Para lluvias con duraciones de 24 horas (1440 min), las magnitudes oscilan entre $41,83 \mathrm{~mm}$ (Puerto Oricao) para un período de retorno de 2 años, y 179,69 mm (La Guitarrita) para un período de retorno de 1000 años. Para escenarios de precipitaciones críticas y concentradas, se observan láminas de lluvias máximas anuales con montos de 121,06 mm, 134,74 mm y 166,20 mm (La Guitarrita), correspondientes a períodos de retorno de 50, 100 y 500 años respectivamente.

Con relación a las intensidades de lluvias máximas anuales, para duraciones de la lluvia de 1 hora, los valores comprenden desde $26,90 \mathrm{~mm} / \mathrm{h}$ (Puerto Oricao) para un período de retorno de 2 años, hasta $111,21 \mathrm{~mm} / \mathrm{h}$ (Puerto Cruz) para un período de retorno de 1000 años. Para escenarios críticos de mayores probabilidades para la activación de procesos erosivos, correspondientes a períodos de retorno de 50, 100 y 500 años con esa misma duración (1 hora), las magnitudes de dichas intensidades se muestran en la figura 5.

Sin embargo, los montos de las intensidades más críticas se registran en los primeros 5 minutos de duración de la lluvia, cuyos valores oscilan entre $69,49 \mathrm{~mm} / \mathrm{h}$ (Puerto Oricao) para un período de retorno de 2 años y 304,02 mm/h (Puerto Cruz) para un período de retorno de 1000 años, así como para escenarios críticos de 50, 100 y 500 años de período de retorno respectivamente.

Mientras que para lluvias de 24 horas de duración, la intensidad abarca desde 1,74 mm/h (2 años de período de retorno) (Puerto Oricao) hasta 7,49 mm/h (1000 años de período de retorno) (La Guitarrita). Los montos estimados para láminas e intensidades máximas, en cada uno de los escenarios discutidos, representan valores críticos o umbrales con un alto potencial para generar procesos erosivos.

La espacialidad de los eventos extremos en la cuenca del río Chichiriviche, muestra una variabilidad que se distingue por la predominancia de mayores montos de láminas máximas anuales e intensidades máximas anuales, en las estaciones representativas de la cuenca baja y franja litoral, en comparación con los que se estimaron para las estaciones de las cuencas media y alta. Esta diferenciación se observa principalmente en los datos estimados para duraciones de la lluvia desde 5 min hasta 180 min y para períodos de retorno desde 10 años hasta 1000 años. Para eventos con 
Figura $\mathrm{N}^{\circ} 4$

Curvas de profundidad - duración - frecuencia (PDF).
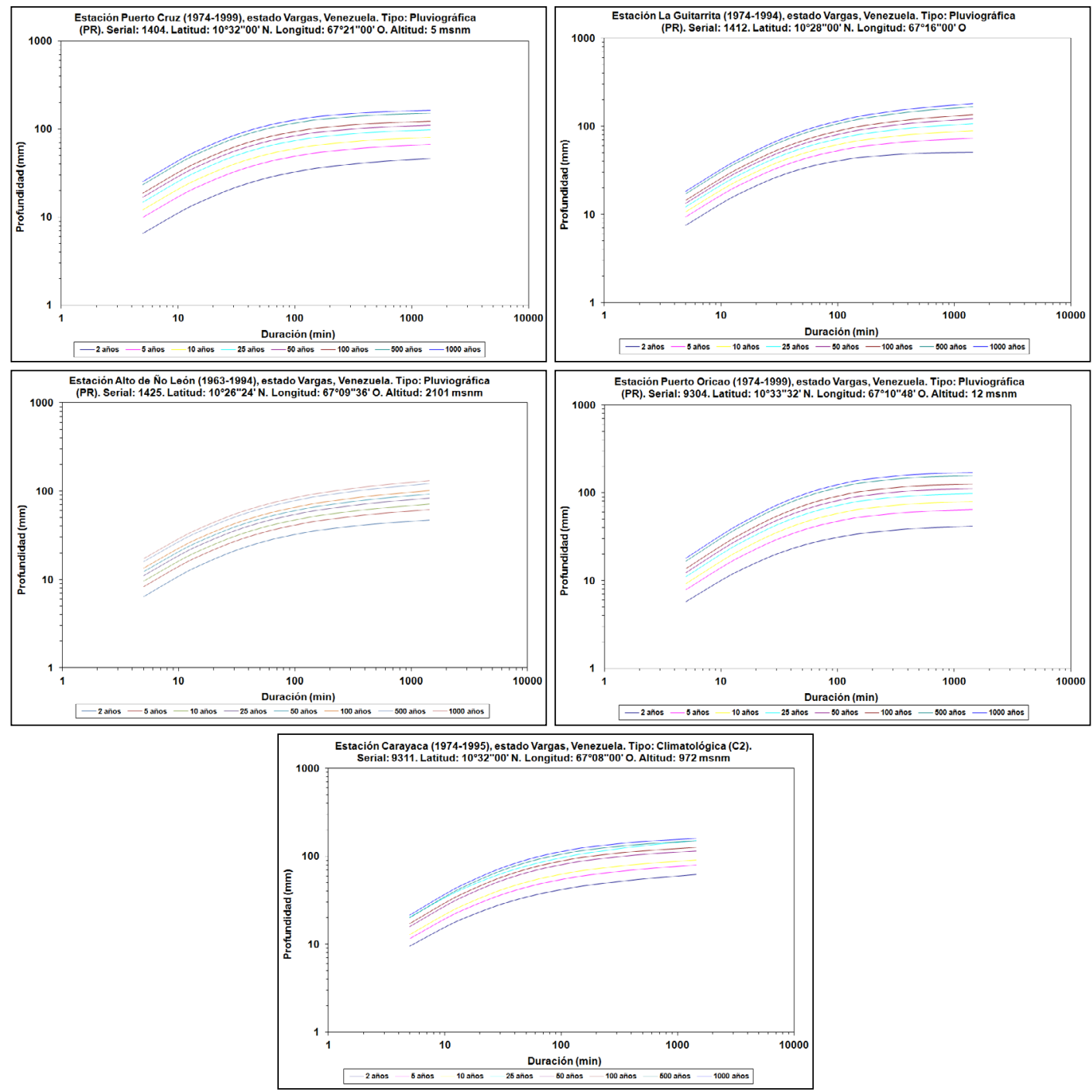

Fuente: Elaboración propia.

duraciones de la lluvia superiores a $180 \mathrm{~min}$ y hasta 24 horas (1440 min) y períodos de retorno más cortos ( 2 y 5 años), los mayores montos se obtuvieron para las estaciones de las cuencas media y alta y, los menores, para la cuenca baja y franja litoral.

Esta distribución indica, una alta probabilidad de ocurrencia de eventos de lluvias extraordinarias con un alto potencial erosivo, sobre el sector comprendido desde la línea de costa hasta la cuenca media, donde precisamente se concentra la mayor superficie intervenida por la actividad agropecuaria. Sin embargo, además del impacto de la lluvia, la variabilidad en cuanto a la intensidad con la que se manifiesten los procesos erosivos en la cuenca, dependerá también de 
otros elementos del medio físico como pendiente, litología, drenaje, cobertura vegetal y nivel de intervención antrópica.

Figura $\mathrm{N}^{\circ} 5$

Curvas de intensidad - duración - frecuencia (IDF).
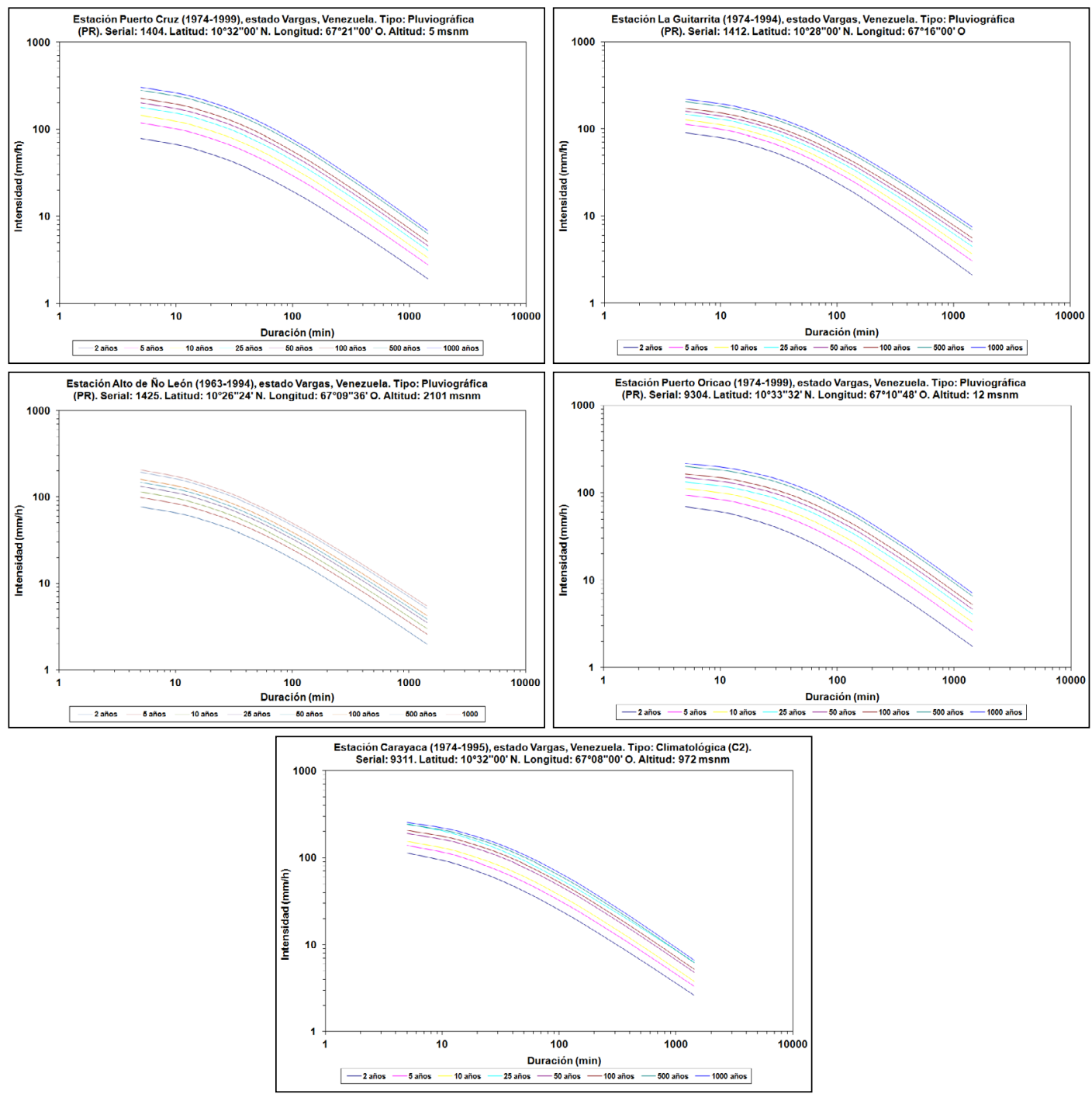

Fuente: Elaboración propia.

\section{Potencial erosivo de las precipitaciones}

Según el IFA, el potencial erosivo de las precipitaciones en la cuenca del río Chichiriviche califica como muy bajo a bajo (figura 6). Los IFA muy bajos se observan en la parte baja de la cuenca y en la franja litoral (estaciones Carayaca, Puerto Oricao, Puerto Chichiriviche y Puerto Cruz), 
precisamente donde ocurren los menores montos pluviométricos anuales; mientras que, para las cuencas media y alta, se presentan los que califican como bajos (La Guitarrita, Hacienda Naranjal, La Peñita y Alto de Ño León), coincidiendo con los sectores donde se registran las mayores precipitaciones anuales en el área de estudio $y$, con los sectores mayoritariamente intervenidos por la actividad agropecuaria, que deja el suelo desnudo y expuesto al impacto de la lluvia.

Figura $\mathrm{N}^{\circ} 6$

Índice de Fournier Anual (IFA).

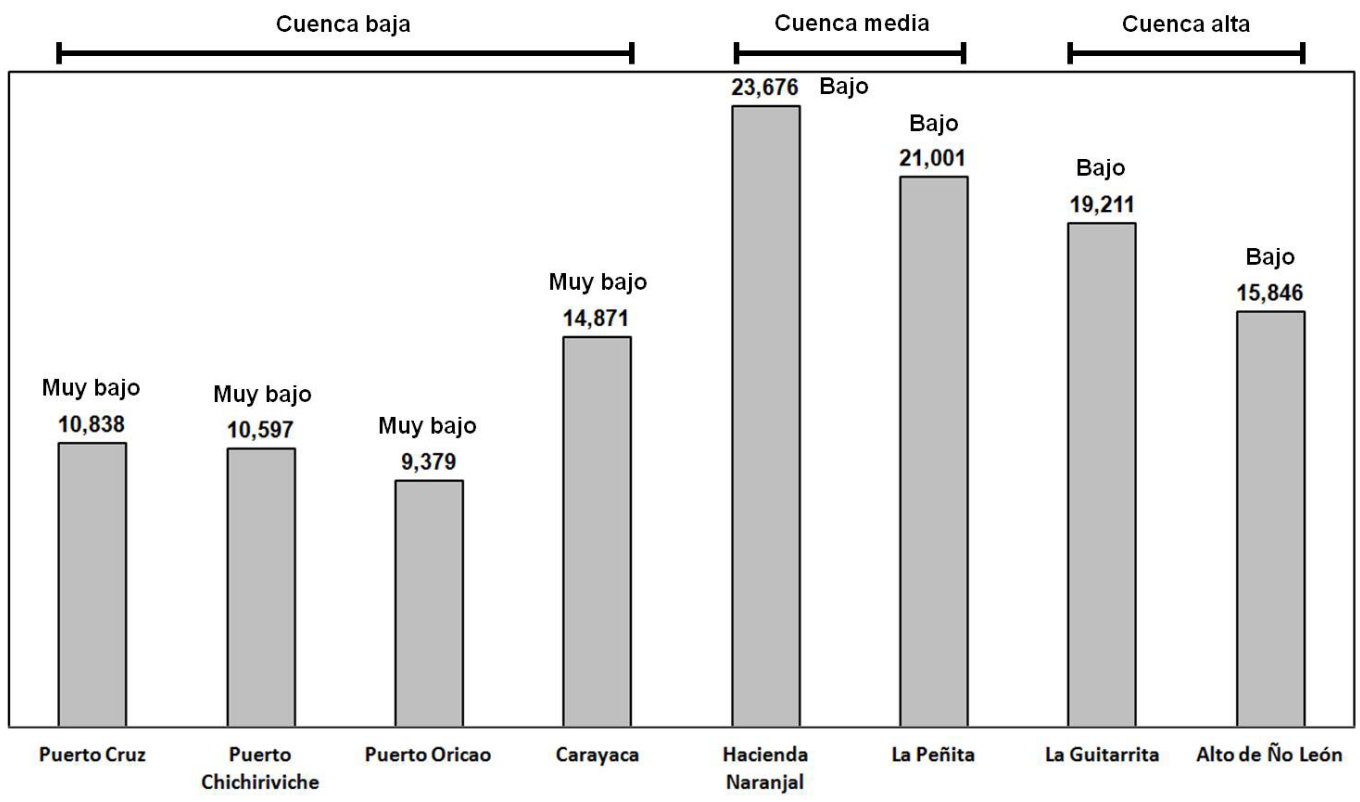

Fuente: Elaboración propia.

De acuerdo con este índice, las precipitaciones como elemento detonante de procesos erosivos en la cuenca, no tienen el suficiente potencial energético para la activación morfogenética, lo cual es contradictorio con las evidencias de campo y el registro histórico de eventos de movimientos en masa para el sector. Probablemente la totalización y promedio de las precipitaciones mensuales (mes más lluvioso), enmascaran eventos extremos significativos, que como episodios aislados y puntuales, si han tenido un potencial morfogenético relevante.

En atención a los IMF1 e IMF2, el potencial de la lluvia tiene un poco más de significado físico, como activador de procesos erosivos. El IMF1 presenta valores entre muy bajo (53,19 en la estación Puerto Oricao) y alto (120,04 en la estación La Guitarrita), y el IMF2 entre bajo (85,69 en la estación Puerto Oricao) y alto (146,55 en la estación La Guitarrita) (figura 7). En particular, el IMF2 funciona como un mejor indicador, ya que se estima para cada año de las series de precipitaciones analizadas, y luego se totalizan. Los IMF1 muy bajos a bajos e IMF2 bajos a moderados, se observan en la cuenca baja y franja litoral, por el contrario, los IMF1 e IMF2 moderados a altos se concentran en los sectores medio y alto de la cuenca. 
Figura $\mathrm{N}^{\circ} 7$

Índice Modificado de Fournier $\left(\mathrm{IMF}_{1}\right.$ e $\left.\mathrm{IMF}_{2}\right)$.

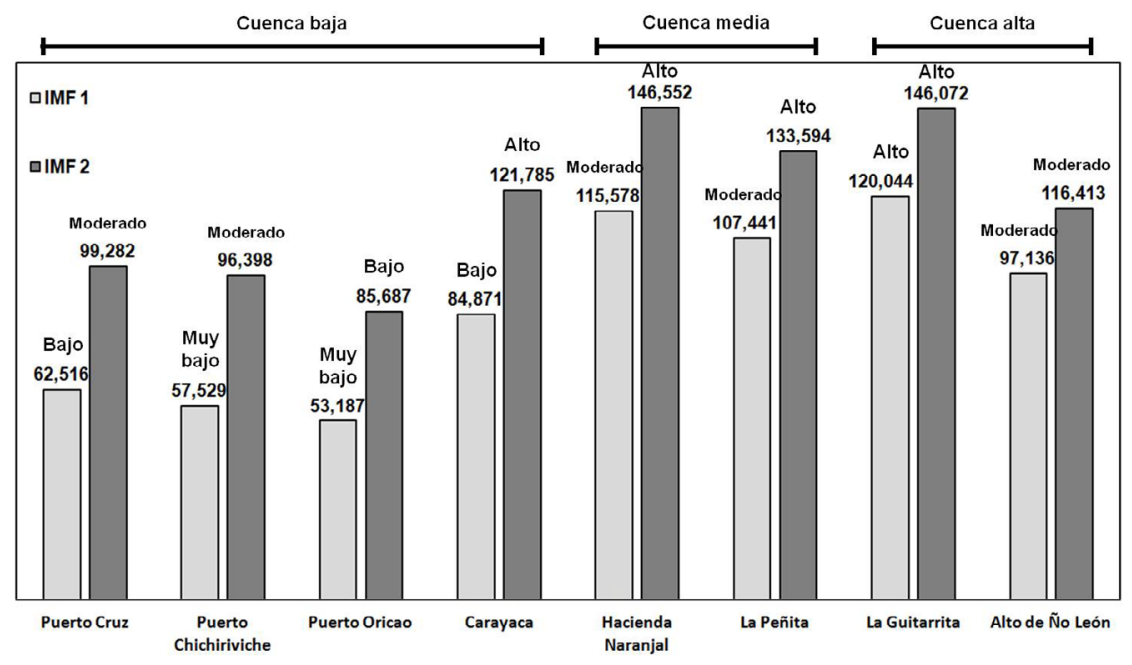

Fuente: Elaboración propia.

Los IMF1 mensuales exhiben una distribución temporal para la parte baja de la cuenca y franja litoral, que se caracteriza por presentar los mayores valores (sin una distribución regular) entre los meses de mayo a diciembre, mientras que para las cuencas media y alta, se perciben entre abril y noviembre (figura 8), lo que significa que en esos períodos las lluvias tienen mayores probabilidades y potenciales para desencadenar procesos erosivos. Sin embargo, en general, las cualificaciones obtenidas a partir de datos mensuales de lluvia para los IMF1 e IMF2, tampoco representan de manera óptima el potencial agresivo de las lluvias en el área, cuando son contrastados con evidencias de activación de fuertes procesos erosivos ocurridos en el sector bajo estudio, detonados por eventos pluviométricos importantes.

Los ICP1 totales definen de manera homogénea un patrón uniforme (valores entre 9,33\% y $10,20 \%$ ) de las lluvias para toda la cuenca (figura 9), con lo cual, las precipitaciones en el área, según este índice, no presentan ningún rasgo de estacionalidad, permitiendo interpretar que, las probabilidades de ocurrencia de un evento erosivo importante es igual para todos los meses del año, escenario que no es real, considerando las condiciones meteorológicas tropicales regulares o "normales" que afectan a la costa venezolana, según las cuales, los eventos pluviométricos más agresivos se presentan, por lo general, en los meses finales del año. Por tales razones, este ICP1 no es un buen indicador del potencial agresivo de las lluvias en el área, puesto que no permite diferenciar o discriminar períodos de agresividad de las precipitaciones.

Por el contrario, los resultados ICP2 mostraron una mayor variabilidad que, determinan patrones de estacionalidad moderada (11,89 como valor más bajo en la estación La Guitarrita) a estacional $(15,83$ como valor más alto en la estación Puerto Chichiriviche) propiamente dicha (figura 9). Según este índice, en cuanto a la distribución espacial del ICP2, se observa una mayor estacionalidad de las lluvias en la cuenca baja y franja litoral y, de manera más moderada para las 
cuencas media y alta, lo cual no es coincidente con los patrones de estacionalidad sugeridos por los pluviogramas de las estaciones consideradas en el estudio.

Figura $\mathrm{N}^{\circ} 8$

Índice Modificado de Fournier Mensual $\left(\mathrm{IMF}_{1}\right)$.

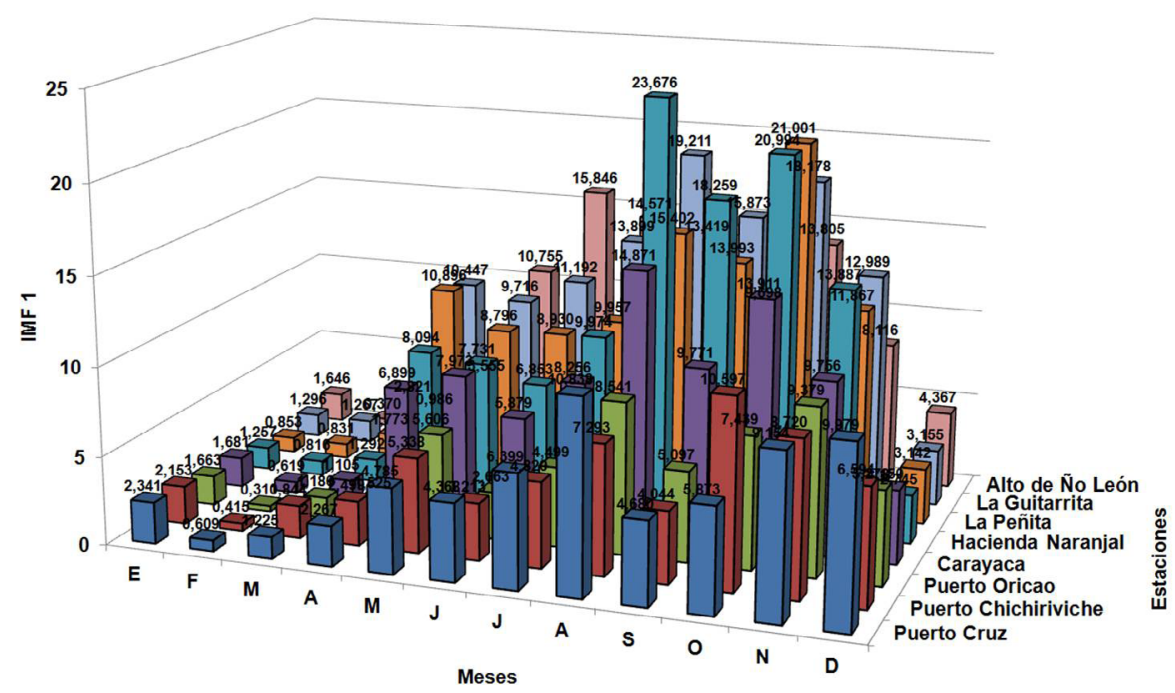

Fuente: Elaboración propia.

Figura $\mathrm{N}^{\circ} 9$

Índice de Concentración de las Precipitaciones $\left(\mathrm{ICP}_{1}\right.$ e ICP $)(\%)$.

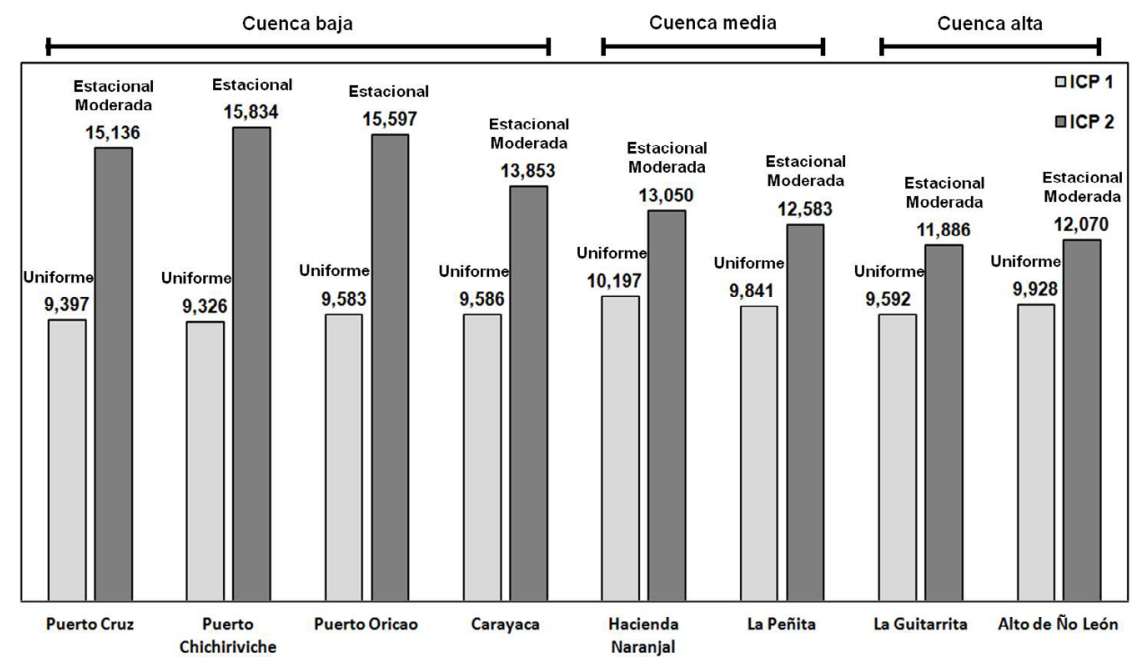

Fuente: Elaboración propia.

Esta discrepancia obedece a que, los ICP2 se calcularon a partir de los totales mensuales de precipitación de cada año de las series analizadas, en los que se puede apreciar, para las estacio- 
nes representativas de la cuenca baja y franja litoral, diferencias muy marcadas entre los montos de precipitación de los meses más lluviosos y los montos de precipitación de los meses más secos, no así, para las estaciones representativas de las cuencas media y alta. En algunos meses de las series, se observan mayores montos de precipitación en las estaciones de la cuenca baja y franja litoral, en contraposición con los registrados en las estaciones de las cuencas media y alta.

Estas características determinan, de manera general, ICP2 con valores más altos (mayor estacionalidad) para cada año de las series temporales consideradas, en las estaciones de la cuenca baja y franja litoral y, valores mucho más bajos en las estaciones de las cuencas media y alta. Por el contrario, los pluviogramas y los ICP1 son construidos y calculados respectivamente, a partir de los promedios mensuales de las series trabajadas, con lo cual, las particularidades de cada año, quedan enmascaradas. Este ICP2 es bastante representativo de la distribución temporal del potencial energético de las lluvias en el área analizada.

Para reforzar la estacionalidad sugerida por los ICP2 anuales o totales, la variabilidad que muestra la distribución temporal de los ICP1 mensuales (figura 10), exhibe un patrón correlativo al observado en la distribución de las lluvias en los pluviogramas. Los ICP1 mensuales oscilan entre valores de $0,04 \%$ (mes de febrero en la estación Alto de Ño León) y 1,92\% (mes de octubre en la estación La Peñita).

Figura $N^{\circ} 10$

Índice de Concentración de las Precipitaciones Mensual (ICP) (\%).

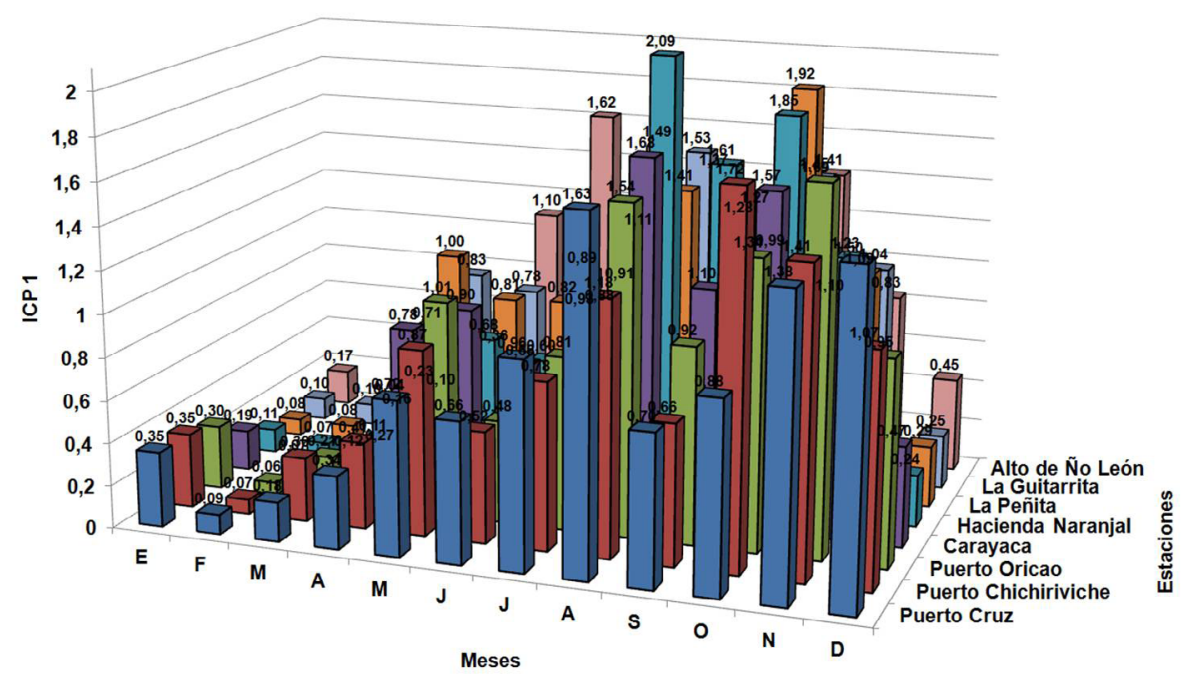

Fuente: Elaboración propia.

Según este índice mensual, las precipitaciones se concentran de manera amplia entre los meses de abril a diciembre y, mayoritariamente, de julio a noviembre. Esto significa que los mayores montos de precipitación en el área, se concentran en un amplio período de 9 meses, considerando las variabilidades temporales entre estaciones $y$, de manera concomitante, se concentran en un período más reducido de 5 meses, lapso éste en el que las lluvias presentan su mayor agresividad como agente detonante de procesos erosivos, tiempo suficiente para la acumulación de 
humedad en el suelo, aunado a la posibilidad de ocurrencia de eventos de lluvias extraordinarias, con la consecuente activación de procesos de remoción en masa.

Los valores correspondientes a los IET1 e IET2 (figura 11), corroboran la baja agresividad o potencial erosivo de las precipitaciones en el área. Los valores de los IET1 oscilan entre 509,71 (estación Oricao) y 1178,54 (estación Hacienda Naranjal), determinando para todas las estaciones un índice de erosividad total bajo; mientras que, los IET2, presentan valores entre 1336,48 (estación Oricao) y 1912,43 (estación Hacienda Naranjal), determinando clasificaciones de erosividad total de baja a moderada.

Figura $\mathrm{N}^{\circ} 11$

Índice de Erosividad Total (IET e IET $_{2}$ ).

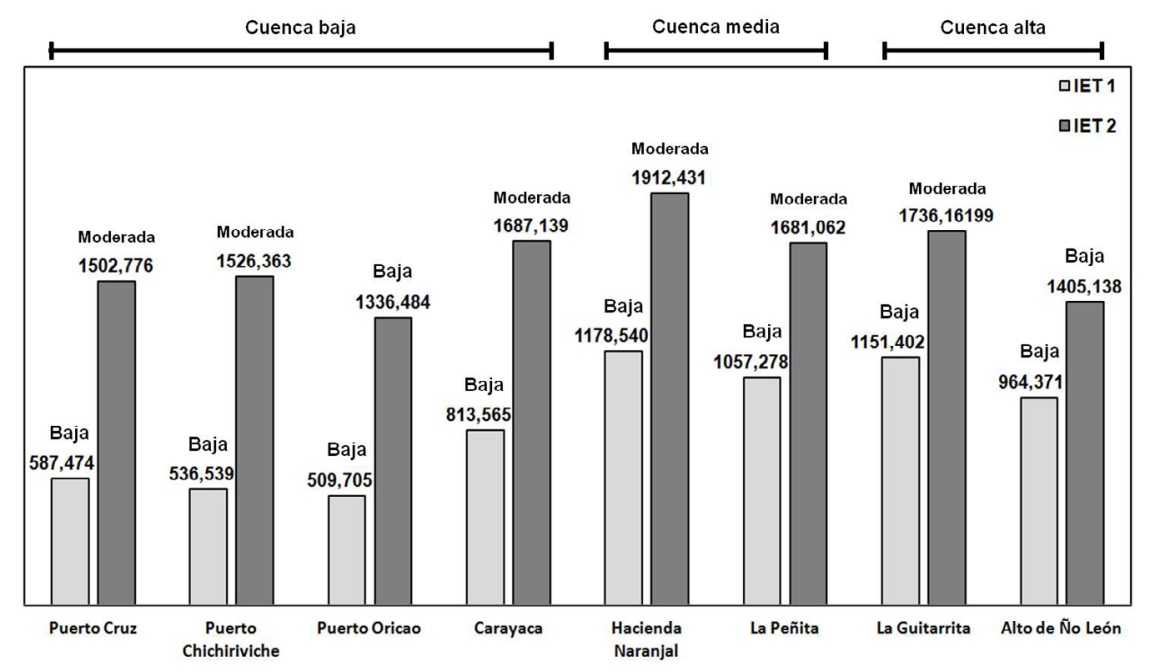

Fuente: Elaboración propia.

Con base en estos resultados, no se aprecia un claro patrón de distribución espacial del potencial erosivo de las lluvias en la cuenca, si no que, por el contrario, se percibe una repartición homogénea, lo cual no se corresponde con lo que ha sido demostrado con los IMF1 mensuales, IMF2, ICP1 mensuales e ICP2. Los IET1 e IET2 no son buenos indicadores del potencial erosivo de las lluvias en el área, puesto que no permiten apreciar su variabilidad espacial.

La estimación del Índice del Potencial Erosivo de las Lluvias o factor R de la Ecuación Universal de Pérdida del Suelo (USLE por sus siglas en inglés) (figura 12), arrojó valores inferiores a $1800 \mathrm{~J} /$ $\mathrm{m} 2 . \mathrm{mm} / \mathrm{h}$ para todas las estaciones incluidas en el análisis, reafirmando el bajo potencial erosivo de las precipitaciones en la cuenca del río Chichiriviche. Los resultados obtenidos oscilan entre 274,23 J/m2.mm/h (estación Hacienda Naranjal) y 1729,53 J/m2.mm/h (estación Carayaca), definiendo potenciales erosivos que clasifican como natural a muy baja respectivamente. Mediante este índice, tampoco es perceptible un claro patrón de distribución espacial del potencial erosivo de las lluvias en la cuenca.

De manera general, algunos de los valores obtenidos (estaciones Carayaca, Puerto Oricao, Alto de Ño León, La Guitarrita y Puerto Cruz) acusan ser bastante homogéneos, sin ninguna varia- 
bilidad contrastante, mientras que los correspondientes a las estaciones Puerto Chichiriviche, La Peñita y Hacienda Naranjal, son muy bajos, lo cual se explica por la carencia de datos estadísticamente suficientes, para profundidades de láminas máximas anuales de precipitación superiores a $12,5 \mathrm{~mm}$, con una duración de $30 \mathrm{~min}$. Este índice, tampoco es un buen indicador de la distribución espacial del potencial energético de las precipitaciones en la cuenca del río Chichiriviche.

Figura $\mathrm{N}^{\circ} 12$

Índice del Potencial Erosivo de las Lluvias (R).

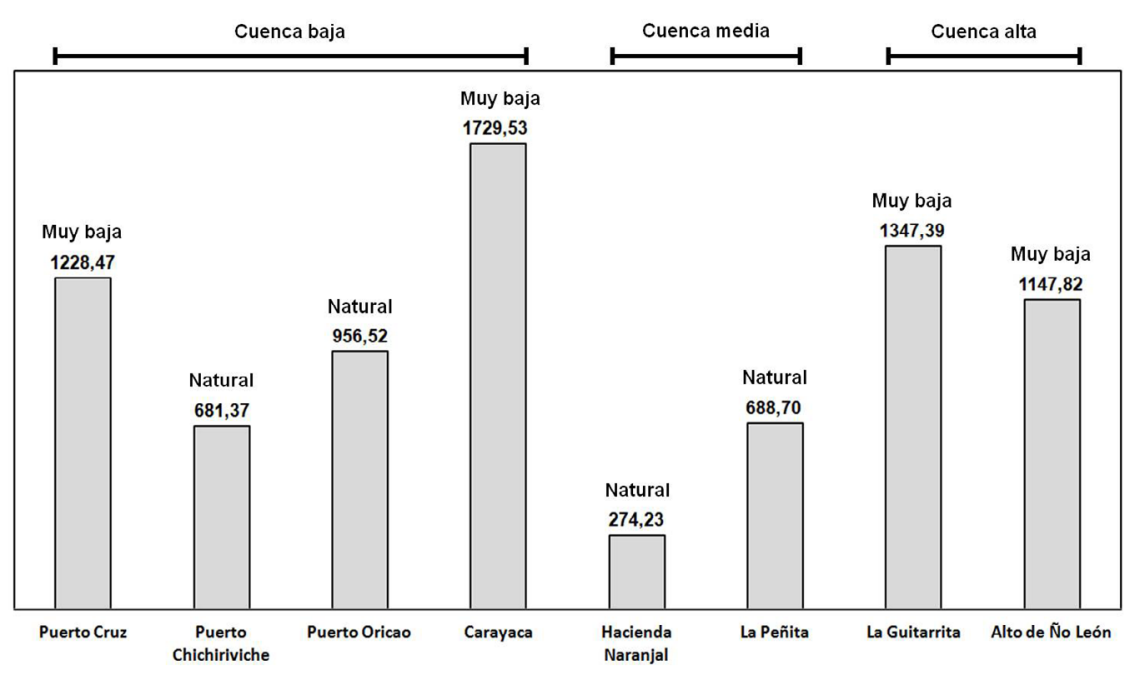

Fuente: Elaboración propia.

\section{Conclusiones}

El análisis espacio-temporal de las precipitaciones en la cuenca del río Chichiriviche evidenció dos patrones pluviométricos contrastantes en cuanto a la proporcionalidad y distribución de los montos mensuales. En la parte baja de la cuenca y franja litoral domina un régimen más uniforme y de bajos montos $y$, en las cuencas media y alta, se exhibe un régimen con una estacionalidad marcada, y montos superiores a los $800 \mathrm{~mm}$.

El análisis de frecuencia de eventos extremos de precipitación, permitió estimar los valores críticos o representativos de los escenarios (duraciones de la lluvia de 1 y 24 horas para períodos de retorno de 50, 100 y 500 años) más significativos, para los cuales pudieran esperarse con alta probabilidad, la activación de procesos erosivos.

En general, los índices considerados para el análisis del potencial erosivo de las precipitaciones en el área, demostraron que el mismo califica de bajo a moderado, a excepción del IMF2, que fue el único en arrojar calificaciones de alto para algunos casos. Los índices de potencial erosivo de las lluvias que mejor funcionan como indicadores de erosividad en la cuenca del río Chichiriviche, son IMF1 (mensuales), IMF2, ICP1 (mensuales) e ICP2. 
El análisis del potencial erosivo de las precipitaciones, empleando como información pluviométrica los totales y promedios mensuales y anuales, enmascaran eventos extraordinarios de lluvia importantes, que como episodios aislados y puntuales, vinculados a situaciones meteorológicas particulares y atípicas, han tenido un potencial relevante en la activación de procesos erosivos. Esta fue la principal limitación en este estudio ya que, el no haber podido disponer de datos pluviométricos horarios y diarios, se dificultó la posibilidad de identificar eventos significativos que, de ser el caso, pudieron haber dado información más confiable y/o precisa sobre el potencial erosivo de las lluvias en el área de estudio.

Para la comprensión integral de la génesis y activación de los procesos erosivos en una cuenca de drenaje montañosa, es necesario complementar este trabajo del potencial erosivo de las precipitaciones como elemento detonante, con el análisis de la susceptibilidad del medio físico frente al impacto de las gotas de lluvia, referido a la dinámica e interacción de otras variables o factores como pendiente, litología, formas de relieve, drenaje, morfometría, cobertura vegetal y uso de la tierra. Esto último permitirá identificar, si el medio físico sólido y biológico de la cuenca determina una alta sensibilidad a la activación de procesos erosivos, aún en presencia de precipitaciones de bajo a moderado potencial energético.

\section{Referencias bibliográficas}

AMEND, S. 1991. Parque Nacional El Ávila (Parques Nacionales y Conservación Ambiental No 2). Stephan y Thora Amend, Caracas, Venezuela.

AMUNDARAY, J. 2000. Aspectos geotécnicos del desastre de Vargas. En: Memorias del XVI Seminario Venezolano de Geotecnia: Calamidades geotécnicas urbanas con visión al siglo XXI, la experiencia para proyectos del futuro. Caracas: Sociedad Venezolana de Geotecnia. P. 261-277.

ANGULO, M. Y BEGUERÍA, S. 2013. Análisis de la erosividad de la lluvia: Procesos, índices y fronteras de conocimiento. Cuaternario y Geomorfología, 27 (1-2), 53-69. Recuperado de https://digital. csic.es/handle/10261/82876

ARISMENDI, J. 2007. Presentación geográfica de las formas de relieve. En: GeoVenezuela 2: Medio físico y recursos ambientales. Caracas: Fundación Empresas Polar. P. 128-182.

ARNOLDUS, H. 1980. An approximation of the rainfall factor in the Universal Soil Loss Equation. En: DE BOODT, M. Y GABRIELS, D. (editores). Assessment of erosion. Chichester: John Wiley \&Sons. P. 127-132.

BALLABIO, C., BORRELLI, P., SPINONI, J., MEUSBURGER, K., MICHAELIDES, S., BEGUERÍA, S., KLIK, A., PETAN, S., JANEČEK, M., OLSEN, P., AALTO, J., LAKATOS, M., RYMSZEWICZ, A., DUMITRESCU, A., PERČEC, M., DIODATO, N., KOSTALOVA, J., ROUSSEVA, S., BANASIK, K., ALEWELL, C. \& PANAGOS, P. 2017. Mapping monthly rainfall erosivity in Europe. Science of the Total Environment, (579), 12981315. doi: 10.1016/j.scitotenv.2016.11.123. Recuperado de https://www.sciencedirect.com/science/ article/pii/S0048969716325773 
CASTELÁN, R., TAMARIZ, V., LINARES, G. Y CRUZ, A. 2014. Agresividad de las precipitaciones en la subcuenca del río San Marcos, Puebla, México. Investigaciones Geográficas, (83), 28-40. doi: 10.14350/rig.33480. Recuperado de https://www.sciencedirect.com/science/article/pii/ S0188461114728031

CHEN, S. \& ZHA, X. 2017. Effects of the ENSO on rainfall erosivity in the Fujian Province of southeast China. Science of the Total Environment, article in press. doi: 10.1016/j.scitotenv.2017.10.090. Recuperado de https://www.sciencedirect.com/science/article/pii/S0048969717327936

DOUKA, M. \& KARACOSTAS, T. 2018. Statistical analyses of extreme rainfall events in Thessaloniki, Greece. Atmospheric Research, (208), 60-77. doi: 10.1016/j.atmosres.2017.08.025. Recuperado de https://www.sciencedirect.com/science/article/pii/s0169809517306154

DUAN, X., GU, Z., LI, Y. \& XU, H. 2016. The spatiotemporal patterns of rainfall erosivity in Yunnan Province, southwest China: An analysis of empirical orthogonal functions. Global and Planetary Change, (144), 82-93. doi: 10.1016/j.gloplacha.2016.07.011. Recuperado de https://www.sciencedirect.com/science/article/pii/S0921818116300042

ESRI, DIGITALGLOBE, GEOEYE, EARTHSTAR GEOGRAPHICS, CNES/AIRBUS DS, USDA, USGS, AEROGRID, IGN, \& THE GIS USER COMMUNITY. 2019. Imagen de satélite del área de la cuenca del río Chichiriviche, estado Vargas, Venezuela, Esri.

FLÓREZ, A. 2018. Lineamientos de gestión territorial en la parroquia Carayaca, estado Vargas: Análisis de conflictos de uso de la tierra. Terra Nueva Etapa, 34 (55), 75-96. doi: 10.35424/rcar. v0i96.186. Recuperado de https://revistasipgh.org/index.php/rcar/article/view/186

FOGHIN, S. 2002. Tiempo y clima en Venezuela: Aproximación a una geografía climática del territorio venezolano (Colección Clase Magistral). Universidad Pedagógica Experimental Libertador, Instituto Pedagógico de Miranda José Manuel Siso Martínez, Subdirección de Investigación y Postgrado, Caracas, Venezuela.

FOURNIER, F. 1960. Climat et erosion: La relation entre l'érosion du sol par l'eau et les précipitations atmosphériques. Presses Universitaires de France, Paris, France.

GAO, M., MO, D. \& WU, X. 2016. Nonstationary modeling of extreme precipitation in China. Atmospheric Research, (182), 1-9. doi: 10.1016/j.atmosres.2016.07.014. Recuperado de https://www. sciencedirect.com/science/article/pii/S0169809516301946

GAO, T., WANG, H. \& ZHOU, T. 2017. Changes of extreme precipitation and nonlinear influence of climate variables over monsoon region in China. Atmospheric Research, (197), 379-389. doi: 10.1016/j.atmosres.2017.07.017. Recuperado de https://www.sciencedirect.com/science/article/ pii/S0169809517301436

GARCÍA-BARRÓN, L., MORALES, J. \& SOUSA, A. 2018. A new methodology for estimating rainfall aggressiveness risk based on daily rainfall records for multi-decennial periods. Science of the To- 
tal Environment, (615), 564-571. doi: 10.1016/j.scitotenv.2017.09.305. Recuperado de https://www. sciencedirect.com/science/article/pii/S0048969717326566

GONZÁLEZ, M. Y CÓRDOVA, J. 2006. Consideraciones sobre la probabilidad de ocurrencia de Iluvias máximas en la zona litoral del norte de Venezuela. En: LÓPEZ, J. Y GARCÍA R. (editores). Los Aludes Torrenciales de Diciembre 1999 en Venezuela. Caracas: Universidad Central de Venezuela, Facultad de Ingeniería, Instituto de Mecánica de Fluidos. P. 98-117.

GOOGLE EARTH. 2019. Imagen de satélite 3D del área de la cuenca del río Chichiriviche, estado Vargas, Venezuela, Google Earth.

GRASES, J., AMUNDARAY, J., MALAVER, A., FELIZIANI, P., FRANCESCHI, L. y RODRÍGUEZ, J. 2000. Emergencia 99: Efectos de las lluvias caídas en Venezuela en diciembre de 1999. Programa de las Naciones Unidas para el Desarrollo, Corporación Andina de Fomento, Caracas, Venezuela.

GU, X., ZHANG, Q., SINGH, V. \& SHI, P. 2017. Nonstationarity in timing of extreme precipitation across China and impact of tropical cyclones. Global and Planetary Change, (149), 153-165. doi: 10.1016/j.gloplacha.2016.12.019. Recuperado de https://www.sciencedirect.com/science/article/ pii/S092181811630323X

GUEVARA, J. 1983. Geografía de las regiones central y capital. Seix Barral, Caracas, Venezuela.

GUEVARA, J. 1987. Métodos de estimación y ajuste de datos climáticos (Colección Monografías 12). Universidad Central de Venezuela, Consejo de Desarrollo Científico y Humanístico, Caracas, Venezuela.

GUMBEL, E. 1941. The return period of flood flows. The Annals of Mathematical Statistics, (12), 163190. Recuperado de https://projecteuclid.org/download/pdf_1/euclid.aoms/1177731747

HAILEGEORGIS, T. \& ALFREDSEN, K. 2017. Analyses of extreme precipitation and runoff events including uncertainties and reliability in design and management of urban water infrastructure. Journal of Hydrology, (544), 290-305. doi: 10.1016/j.jhydrol.2016.11.037. Recuperado de https:// www.sciencedirect.com/science/article/pii/S0022169416307430

HOOMEHR, S., SCHWARTZ, J. \& YODER, D. 2016. Potential changes in rainfall erosivity under GCM climate change scenarios for the southern Appalachian region, USA. Catena, (136), 141-151. doi: 10.1016/j.catena.2015.01.012. Recuperado de https://www.sciencedirect.com/science/article/abs/ pii/S034181621500017X

KITE, G. 1977. Frequency and risk analysis in hydrology. Water Resources Publications, Fort Collins, Colorado, USA.

LIMSAKUL, A. \& SINGHRUCK, P. 2016. Long-term trends and variability of total and extreme precipitation in Thailand. Atmospheric Research, (169), 301-317. doi: 10.1016/j.atmosres.2015.10.015. Recuperado de https://www.sciencedirect.com/science/article/pii/S0169809515003361 
MESHESHA, D., TSUNEKAWA, A., TSUBO, M., HAREGEWEYN, N. \& TEGEGNE, F. 2016. Evaluation of kinetic energy and erosivity potential of simulated rainfall using Laser Precipitation Monitor. Catena, (137), 237-243. doi: 10.1016/j.catena.2015.09.017. Recuperado de https://www.sciencedirect. com/science/article/abs/pii/S034181621530117X

MINISTERIO DEL AMBIENTE DE LOS RECURSOS NATURALES RENOVABLES. 1983. Sistemas Ambientales Venezolanos (Serie de Informes Técnicos Proyecto VEN/79/001). Caracas, Venezuela.

MINISTERIO DEL PODER POPULAR PARA EL AMBIENTE, DIRECCIÓN GENERAL DE CUENCAS, DIRECCIÓN DE HIDROLOGÍA Y METEOROLOGÍA. 2011. Datos climatológicos: Puerto Cruz, La Guitarrita, La Peñita, Alto de Ño León, Puerto Oricao, Carayaca, Puerto Chichiriviche y Hacienda Naranjal. Datos no publicados. Caracas, Venezuela.

MINISTERIO DEL PODER POPULAR PARA EL AMBIENTE, DIRECCIÓN GENERAL DE CUENCAS, DIRECCIÓN DE HIDROLOGÍA Y METEOROLOGÍA. 2015. Datos climatológicos: Puerto Cruz, La Guitarrita, La Peñita, Alto de Ño León, Puerto Oricao, Carayaca, Puerto Chichiriviche y Hacienda Naranjal. Datos no publicados. Caracas, Venezuela.

PAKALIDOU, N. \& KARACOSTA, P. 2018. Study of very long-period extreme precipitation records in Thessaloniki, Greece. Atmospheric Research, (208), 106-115. doi: 10.1016/j.atmosres.2017.07.029. Recuperado de https://www.sciencedirect.com/science/article/pii/S0169809517306129

PANAGOS, P., BALLABIO, C., BORRELLI, P. \& MEUSBURGER, K. 2016. Spatio-temporal analysis of rainfall erosivity and erosivity density in Greece. Catena, (137), 161-172. doi:10.1016/j.catena.2015.09.015. Recuperado de https://www.sciencedirect.com/science/article/pii/S0341816215301156

PANAGOS, P., BALLABIO, C., MEUSBURGER, K., SPINONI, J., ALEWELL, C. \& BORRELLI, P. 2017. Towards estimates of future rainfall erosivity in Europe based on REDES and WorldClim datasets. Journal of Hydrology, (5348), 251-262. doi: 10.1016/j.jhydrol.2017.03.006. Recuperado de https:// www.sciencedirect.com/science/article/pii/S0022169417301439

PETRÓlEOS DE VENEZUELA, S. A. 1993. Atlas Imagen de Venezuela: Una visión desde el espacio. Caracas, Venezuela.

PONVERT, D., LAU, A. Y BALAMASEDA, C. 2007. La vulnerabilidad del sector agrícola frente a los desastres: Reflexiones generales. Zonas Áridas, 11 (1), 174-194. doi: 10.21704/za.v11i1.214. Recuperado de http://revistas.lamolina.edu.pe/index.php/rza/article/view/214

QIN, W., GUO, Q., ZUO, C., SHAN, Z., MA, L. Y SUN, G. 2016. Spatial distribution and temporal trends of rainfall erosivity in mainland China for 1951-2010. Catena, (147), 177-186. doi: 10.1016/j. catena.2016.07.006. Recuperado de https://www.sciencedirect.com/science/article/abs/pii/ S034181621630265X

SANTOS, M., FRAGOSO, M. \& SANTOS, J. 2017. Regionalization and susceptibility assessment to daily precipitation extremes in mainland Portugal. Applied Geography, (86), 128-138. doi: 10.1016/j. 
apgeog.2017.06.020. Recuperado de https://www.sciencedirect.com/science/article/abs/pii/ S0143622817301406

STEYERMARK, J. Y HUBER, O. 1978. Flora del Ávila: Flora y vegetación de las montañas del Ávila, de la Silla y del Naiguatá. Sociedad Venezolana de Ciencias Naturales, Völlmer Foundation, Ministerio del Ambiente y de los Recursos Naturales Renovables, Caracas, Venezuela.

U. S. WATER RESOURCES COUNCIL. 1981. Guidelines for determining flood flow frequency (Bulletin 17B). Office of Water Data Coordination, U. S. Geological Survey, Reston, USA.

URBANI, F. Y RODRÍGUEZ, J. 2004. Atlas geológico de la Cordillera de la Costa, Venezuela. Escuela de Geología, Minas y Geofísica de la Universidad Central de Venezuela, Fundación Venezolana de Investigaciones Sismológicas, Fundación Geos, Caracas, Venezuela.

URBANI, F., RODRÍGUEZ, J., BARBOZA, L., RODRÍGUEZ, S., CANO, V., MELO, L., CASTILLO, A., SUÁREZ, J., VIVAS, V. Y FOURNIER, H. 2006. Geología del estado Vargas, Venezuela. En: LÓPEZ, J. y GARCÍA, R. (editores). Los aludes torrenciales de diciembre 1999 en Venezuela. Instituto de Mecánica de Fluidos, Facultad de Ingeniería, Universidad Central de Venezuela, Caracas, Venezuela. P. 133-156.

VILA, P. 1960. Geografía de Venezuela 1: El territorio nacional y su ambiente físico. Ministerio de Educación, Caracas, Venezuela.

WANG, X., HOU, X. \& WANG, Y. 2017. Spatiotemporal variations and regional differences of extreme precipitation events in the Coastal area of China from 1961 to 2014. Atmospheric Research, (197), 94-104. doi: 10.1016/j.atmosres.2017.06.022. Recuperado de https://www.sciencedirect.com/ science/article/pii/S0169809517300236

WENZEL, H. 1982. Rainfall for urban stormwater design. En: Urban stormwater hydrology (Serie Water Resources Monograph 7). American Geophysical Union, Washington, USA. P. 35-67.

WISCHMEIER, W. 1959. A rainfall erosion index for a Universal Soil-Loss Equation. Soil Science Society Proceedings, (23), 246-249. doi: 10.2136/sssaj1959.03615995002300030027x. Recuperado de https://acsess.onlinelibrary.wiley.com/doi/epdf/10.2136/sssaj1959.03615995002300030027x

XIE, Y., YIN, S., LIU, B., NEARING, M. \& ZHAO, Y. 2016. Models for estimating daily rainfall erosivity in China. Journal of Hydrology, (535), 547-558. doi: 10.1016/j.jhydrol.2016.02.020. Recuperado de https://www.sciencedirect.com/science/article/pii/S0022169416300531 
